\title{
Value-driven attentional capture is modulated by the contents of working memory: An EEG study
}

\author{
T. Hinault ${ }^{1}$ (D) $\cdot$ K. J. Blacker ${ }^{1,2} \cdot$ M. Gormley ${ }^{1}$ • B. A. Anderson ${ }^{3} \cdot$ S. M. Courtney ${ }^{1,4,5}$
}

Published online: 20 November 2018

(C) Psychonomic Society, Inc. 2018

\begin{abstract}
Attention and working memory (WM) have previously been shown to interact closely when sensory information is being maintained. However, when non-sensory information is maintained in WM, the relationship between WM and sensory attention may be less strong. In the current study, we used electroencephalography to evaluate whether value-driven attentional capture (i.e., allocation of attention to a task-irrelevant feature previously associated with a reward) and its effects on either sensory or non-sensory WM performance might be greater than the effects of salient, non-reward-associated stimuli. In a training phase, 19 participants learned to associate a color with reward. Then, participants were presented with squares and encoded their locations into WM. Participants were instructed to convert the spatial locations either to another type of sensory representation or to an abstract, relational type of representation. During the WM delay period, task-irrelevant distractors, either previously-rewarded or non-rewarded, were presented, with a novel color distractor in the other hemifield. The results revealed lower alpha power and larger N2pc amplitude over posterior electrode sides contralateral to the previously rewarded color, compared to ipsilateral. These effects were mainly found during relational WM, compared to sensory WM, and only for the previously rewarded distractor color, compared to a previous non-rewarded target color or novel color. These effects were associated with modulations of WM performance. These results appear to reflect less capture of attention during maintenance of specific location information, and suggest that value-driven attentional capture can be mitigated as a function of the type of information maintained in WM.
\end{abstract}

Keywords EEG $\cdot$ Working memory $\cdot$ Alpha power $\cdot$ Value $\cdot$ Attentional capture

\section{Introduction}

Active maintenance and manipulation of task-relevant information in working memory (WM), together with suppression of potential interference from competing task-irrelevant

\section{T. Hinault}

thomas.hinault@jhu.edu

1 Department of Psychological and Brain Sciences, Johns Hopkins University, 143 Ames Hall, 3400 N. Charles Street, Baltimore, MD 21218, USA

2 The Henry M. Jackson Foundation for the Advancement of Military Medicine, Inc, Bethesda, MD, USA

3 Department of Psychological and Brain Sciences, Texas A\&M University, College Station, TX 4235, USA

4 Department of Neuroscience, Johns Hopkins University, Baltimore, MD, USA

5 F.M. Kirby Research Center, Kennedy Krieger Institute, Baltimore, MD, USA information, are central for decision making and goaldirected behaviors. In addition to the prefrontal cortex, WM processes often recruit posterior sensory regions to help maintain some kinds of information when that information is no longer present perceptually. The capture of attention by salient yet irrelevant stimuli strongly influences the efficacy of the maintenance of task-relevant sensory information in WM (e.g., Awh et al., 1998; Jonides \& Yantis, 1988). Conversely, the maintenance of sensory information in WM can also bias sensory selective attention and attention capture (e.g., Makovski et al., 2011; Sala \& Courtney, 2009; Unsworth \& Robinson, 2016; see Fougnie, 2008, for a review). WM processes, however, can also transform relevant information into potentially more stable, non-sensory, representations that are adapted to behavioral goals (e.g., Andersen et al., 1997; Courtney, 2004; D'Esposito et al., 2000). This transformation raises the question of how attentional processes, such as the capture of attention, might be differentially modulated by the sensory versus non-sensory nature of current WM representations. Similarly, the efficacy of WM might be modulated by 
the nature of the attentional processes involved, such as the degree to which attentional capture is driven by stimulus salience. Recent research has shown that learned associations between stimulus features and reward can result in later attentional capture that is distinct from attentional capture due to perceptual salience, a process referred to as value-driven attentional capture (VDAC, see Anderson, 2013, 2016, for reviews). Here, we investigated the nature of the relationship between attention and WM by using electroencephalography (EEG) to evaluate whether VDAC is modulated by or differentially affects the maintenance of different types of information in WM.

The WM tasks used in the current study are based on previous work demonstrating that the cognitive and neural systems engaged during maintenance and manipulation of information in WM differ as a function of the type of information being processed (e.g., Blacker \& Courtney, 2016; Blacker et al., 2016; Ikkai et al., 2014). The authors used a visual WM task that involved maintaining either concrete spatial coordinates or abstract relational information (i.e., spatial relationships between objects). For example, in one version of the tasks used in these experiments, participants were instructed to remember the locations of two stimuli on the screen. After an initial delay, a cue indicated whether the trial was a Relation or a Location trial. For Location (i.e., sensory) trials, participants were instructed to imagine a line that connected the two stimuli and maintain the location of that line in WM. For Relation (i.e., abstract) trials, participants were instructed to remember the vertical position of one stimulus relative to the other (i.e., which stimulus is above the other). Previous functional magnetic resonance imaging (fMRI) work has revealed distinct neural correlates for Relation and Location trials, with larger activation of the visual cortex, posterior parietal cortex, and prefrontal regions for Locations relative to Relation trials, while Relation trials showed larger activation in parahippocampal gyrus and precuneus compared to Location trials. There were no differences in brain activation in areas traditionally associated with language processing. In addition, the introduction of a verbal load showed no interaction with the trial type, suggesting that the results did not reflect a differential reliance on verbal strategy (Ikkai et al., 2014). These results indicate that these location and relation WM tasks involve maintenance of different types of information, and the distinction is not one of verbal versus visual information. While uncertainty remains regarding the nature of WM representations during both Location and Relation tasks, as has been discussed previously in the sensory WM literature (e.g., Miller 1956; Postle \& Hamidi, 2007), these results indicate that Location and Relation WM tasks involve maintenance of different types of information, and the distinction is not one of verbal versus visual information. Relational WM likely involves the conversion of sensory information to new, abstract representations, and this conversion appears to rely on the efficient suppression of sensory regions, as might be expected since maintained sensory representation of the sample stimulus might interfere with accurate performance in the Relational WM task but not in the Location WM task.

Previous electroencephalography (EEG) studies with similar tasks revealed that, following the cue indicating whether the trial was a Relation or a Location trial, conversion and maintenance of an abstract representation (i.e., in Relation trials) resulted in increased alpha $(8-13 \mathrm{~Hz})$ power over posterior electrode sites compared to maintenance of a sensory representation (i.e., in Location trials). Alpha modulations during maintenance of abstract information in WM were interpreted as reflecting the suppression of posterior sensory representations that were irrelevant to the current task, consistent with previous theories of the role of alpha oscillatory activity in selective attention (e.g., Jensen et al., 2002; Klimesch et al., 1999, 2007, 2012). These results might suggest that WM for abstract relationships might be less susceptible to interference from attention to irrelevant stimuli. However, the role of oscillatory activity in attention, its role in WM, and the interaction between attention and WM content are still poorly understood.

One way in which WM and attention interact is the protection of relevant information in WM by inhibiting the processing of irrelevant, distracting stimuli via selective attention. The success of this inhibitory process depends on both the physical salience and previous task-relevance of the stimuli. In addition to previous research on location and relation WM tasks, the current study also draws on previous research demonstrating that the previous value of a stimulus can influence the allocation of attention, even when this stimulus is no longer relevant (Anderson et al., 2011, 2016, 2017; Anderson \& Halpern, 2017; Rutherford et al., 2010). To study VDAC, previous work relied on a paradigm including a training and a test phase. During the training phase, participants implicitly associated a certain color with a reward, and were asked to report the orientation of a line (i.e., horizontal or vertical) within a target shape (i.e., a red or a green circle). Participants received money when they reported the orientation of the line in a circle of a specific target color (e.g., red). In a subsequent test phase, participants searched for a uniquely shaped singleton (i.e., a circle) among non-target shapes (i.e., diamonds), with color irrelevant to the current task demand. Performance was found to be slower when the task-irrelevant stimulus feature (i.e., the color of a non-target shape), previously associated with a reward, was simultaneously presented with the target.

EEG studies investigated the time-course of VDAC. Using the VDAC paradigm, MacLean and Giesbrecht (2015) showed modulations of the P1 event-related potential (ERP) component (i.e., positive deflection over posterior-occipital sites around $100 \mathrm{~ms}$ after stimulus onset). P1 amplitude was larger on electrode sites contralateral to task-irrelevant yet 
value-associated features, in contrast with ipsilateral sites. This modulation is in line with selective attentional capture by value-associated properties of stimuli during the early stages of visual processing. Moreover, Qi et al. (2013) showed modulations of the N2pc component (i.e., parieto-occipital negative deflection contralateral to the location of an attended stimulus between $200 \mathrm{~ms}$ and $300 \mathrm{~ms}$ after stimulus onset; Eimer, 1996; Hickey et al., 2006; Luck \& Hillyard, 1994; Woodman \& Luck, 1999). Indeed, amplitudes were significantly more negative on electrodes sites contralateral to the presentation of a value-associated distractor when compared to distractors that were not previously associated with a reward (see also Itthipuripat et al., 2015). N2pc amplitude was negatively correlated with individual differences in performance during the reward task, being more negative in people with stronger value learning (Qi et al., 2013). Although some studies associated this component with distractor filtering (e.g., Luck \& Ford, 1998; Luck \& Hillyard, 1994; Luck, 2012; Noonan et al., 2016), the majority of studies interpreted the N2pc component as reflecting attentional orienting (e.g., Kumar et al., 2009, 2016), and the capture of attention by physically salient yet task-irrelevant stimuli (e.g., Hickey et al., 2006; Kiss et al., 2012; Qi et al., 2013; Mazza et al., 2009; Sawaki et al., 2013; Töllner et al., 2012; Wykowska and Schubö, 2009; see Eimer \& Kiss, 2008, for a review). Thus, VDAC appears to affect both early visual processing and attentional filtering or orienting, and thus could interact with both sensory and non-sensory WM, but potentially in different ways or to different degrees.

Attentional processing and attentional capture have also been associated with modulations of alpha oscillatory activity. Following a relevant probe, decreased alpha power was observed over sensory regions processing the target, compared to regions processing the distractors, therefore facilitating the processing of the target information (e.g., Capotosto et al., 2009; Händel et al., 2011; Jensen et al., 2012; Keehn et al., 2017; Klimesch, 2012; van Diepen et al., 2016). Salient yet irrelevant events have also been associated with decreased alpha power, in line with attentional capture (e.g., Keehn et al., 2017). In the present study, we hypothesized that VAC, and the associated neural correlates reflected in oscillatory activity and ERPs, might also affect WM maintenance. Given that our previous research (Blacker et al., 2016; Ikkai et al., 2014) indicated that alpha power is also modulated by the type of information maintained during WM, attentional capture could differentially influence the maintenance of sensory and abstract information in WM. As the maintenance of relational information in WM does not require the highly selective allocation of spatial attention that location WM does, we expected relation WM to be more vulnerable to attention capture by salient yet irrelevant information, and perhaps more vulnerable to VDAC.
Here, we used EEG to measure the time course of neural activity during sensory and abstract WM maintenance, and in response to the presentation of previously relevant distractors during the WM delay. The main goal of our study was to investigate whether the neural correlates (i.e., ERPs, and oscillatory activity) of VDAC and its effects on WM differ as a function of the maintenance of a sensory representation versus an abstract representation in WM. We expected to observe changes in ERP amplitude and alpha power when an irrelevant but previously rewarded stimulus feature was displayed, compared to a non-rewarded or a novel stimulus, and that this effect would be influenced by the type of information maintained in WM. Modulations of N2pc and alpha power were expected to be larger for Relation than Location trials for a previously rewarded stimulus, relative to a novel or a previously relevant but non-rewarded stimulus. The present study offers valuable insights into: (a) the interactions between attentional capture and WM maintenance, (b) the type of information in WM that is most vulnerable to influence by attentional capture, and (c) the time course of such capture.

\section{Methods}

Participants Nineteen volunteers participated in this experiment (14 females, mean age $20.8 \pm 2.6$ years) and were compensated monetarily. All participants were right-handed, and reported normal or corrected-to-normal vision. All participants gave written informed consent approved by the Institutional Review Board of Johns Hopkins University. Twenty-six participants were originally recruited. Seven participants were excluded from analyses due to excessive EEG artifacts $(<70 \%$ of epochs after preprocessing), and/or incorrect trials ( $<60 \%$ accuracy on the WM task), and/or technical difficulties. The sample size used in this experiment was based on a power analysis conducted in G*Power 3.1 (Faul et al., 2007). Assuming an effect size of Cohen's $f=1.28$ (derived from relevant previously published studies; e.g., Qi et al., 2013), an alpha of .05, and one group, we determined that a total sample size of 19 participants would provide $95 \%$ power to detect the effects.

Experimental paradigm Experimental stimuli were controlled by MATLAB (The MathWorks, Natick, MA, USA) using PsychToolbox extensions (Brainard, 1997; Pelli, 1997), and displayed on a back-projector screen using a waveguide with the projector outside the shielded room. A photodiode was used to measure the delay in stimulus display. Participants were seated $108 \mathrm{~cm}$ away from the screen, and given a Logitech game controller to enter responses.

During the training phase (Fig. 1), participants performed a visual search task adapted from Anderson et al. (2011). The fixation display consisted of a central black fixation cross 


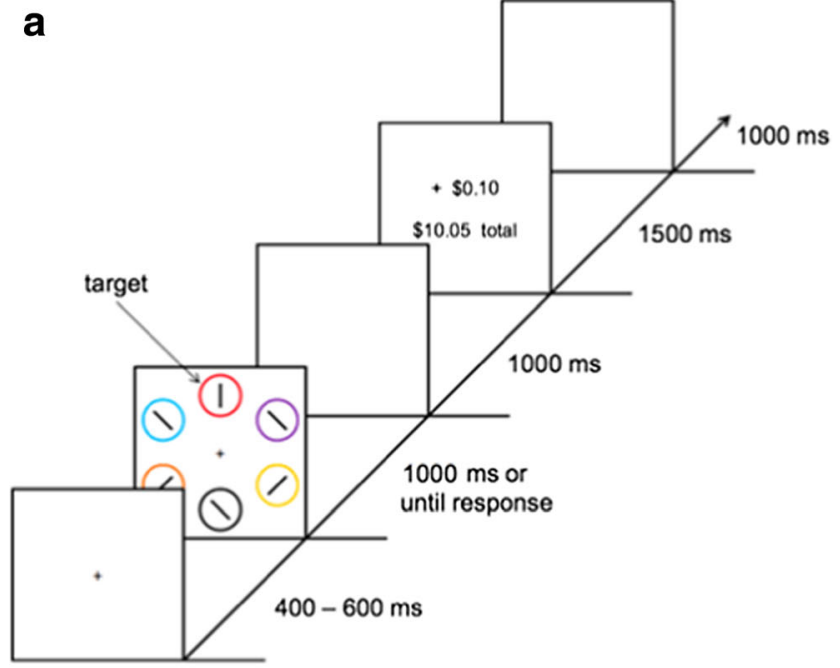

b

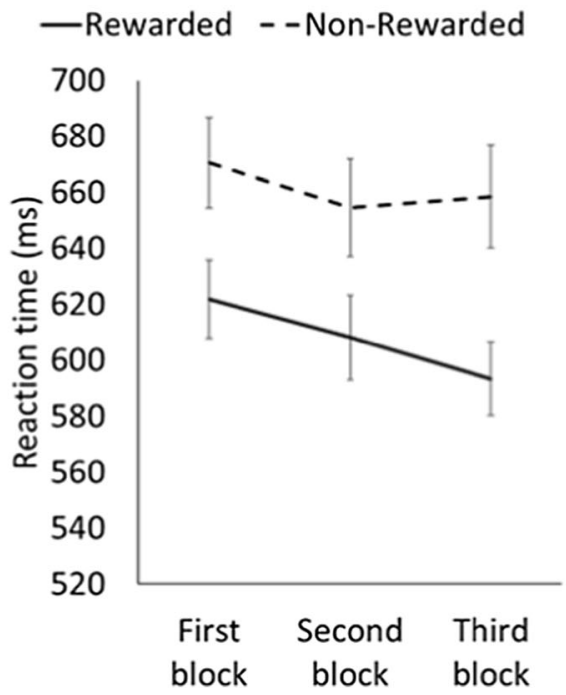

Fig. 1 A, Trial schematics showing the sequence of events in the reward training task. Participants had to indicate the orientation of the line within the target colored circle (green or red) and were monetarily rewarded for one of the two colors when the response was correct. B, Differences between responses to rewarded and non-rewarded targets during the reward training task, as a function of the block of trials

$\left(0.7^{\circ} \times 0.7^{\circ}\right.$ visual angle $)$, presented against a white background . The search display consisted of six circles $\left(2.8^{\circ} \times 2.8^{\circ}\right)$, each equidistant $\left(4.5^{\circ}\right)$ from the fixation cross. Six circles with different colors were presented in the search display (colors: red/ green, pink, orange, yellow, light blue, and black). Targets were defined as a red or a green circle, only one of which was presented on each trial. Inside the target, a line was oriented either vertically or horizontally. Participants were asked to report the orientation of the line within the target circle by pressing one of two horizontally aligned buttons on the controller. Orientationto-response mapping was counterbalanced across participants. The training task was performed under time pressure, with trials terminating after 1,000 ms. Correct and fast responses (less than
$1,000 \mathrm{~ms}$ ) were followed by reward feedback. The feedback display informed participants of the reward on the current trial, as well as total reward accumulated thus far. For one of the color targets, participants received $\$ 0.25$ on $80 \%$ of the correct trials and $\$ 0.10$ on $20 \%$ of the correct trials. Participants were clearly told that their additional pay was determined by their performance in the task. The rewarded target was red for half of the participants, and green for the other half. The unrewarded color was always followed by $\$ 0.00$ and the total earned from previous trials. The first training block consisted of 240 trials, in a single session. Interspersed within the test phase were two additional training blocks of 60 trials each. Upon completion of the experiment, participants were given the cumulative monetary reward they had earned.

During the WM test phase, participants performed a variant of the spatial WM tasks previously used by our group (Blacker \& Courtney, 2016; Blacker et al., 2016; Ikkai et al., 2014). As shown in Fig. 2A, a trial began with a black fixation cross $\left(0.1^{\circ}\right.$ of visual angle) appearing in the center of a gray background for $700 \mathrm{~ms}$. Then, a 700-ms cue indicated whether participants were to remember a particular location (i.e., Location trial, cued by the word "Location") or the relative spatial relationship between the items (i.e., Relation trial, cued by the word "Relation"). A sample array was then presented for $500 \mathrm{~ms}$, which contained four black, gray, or white squares (i.e., two squares of different luminances in each hemifield, each square subtending $0.3^{\circ}$ of visual angle). After a jittered 1,500-2,000 ms Delay 1 period, six colored distractors, three in each hemifield, were displayed for $100 \mathrm{~ms}$. The distractors in each hemifield were either red (i.e., previously rewarded/ non-rewarded color), green (i.e., previously rewarded/nonrewarded color), or blue (i.e., novel color), with only one color per hemifield. The mapping of red or green stimuli to be either the rewarded or non-rewarded color in the training task was counterbalanced across participants. The novel color was blue for every participant. The novel color was always presented in one hemifield together with either the previously rewarded or the non-rewarded color in the other hemifield. The distractors were followed by a jittered 1,400-1,900 ms Delay 2 period. The display duration of the two delay periods plus the presentation of the distractors always equaled 3,500 ms. Following the delay period, two squares in the same hemifield were presented as the test array for 1,200 ms, during which the participant entered a response. The test array was followed by a $100-\mathrm{ms}$ feedback period where the word "correct," "incorrect," or "too slow" was displayed. In some previous versions of this task, the trial type was indicated following the WM sample array, and in other previous studies, the trial type instruction was presented at the beginning of the trial. Those studies found similar dissociations between Location and Relation WM. In the current study, the Location/Relation trial type instruction was presented before the presentation of the WM sample array in order to add the distractor phase and 
a

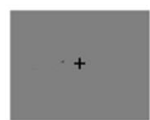

$700 \mathrm{~ms}$

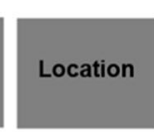

$700 \mathrm{~ms}$

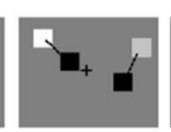

$500 \mathrm{~ms}$
Delay 1

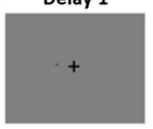

$1500-2000 \mathrm{~ms}$

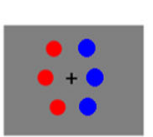

$100 \mathrm{~ms}$
Delay 2

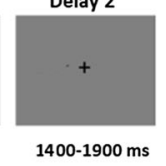

MATCH

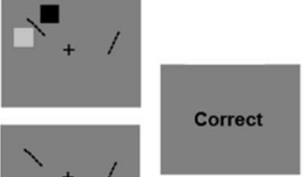

$100 \mathrm{~ms}$
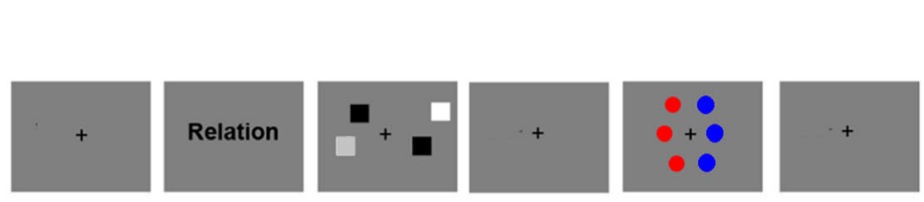

NON-MATCH

$1200 \mathrm{~ms}$

MATCH
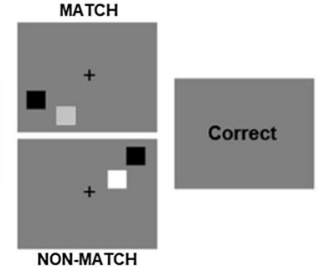

C

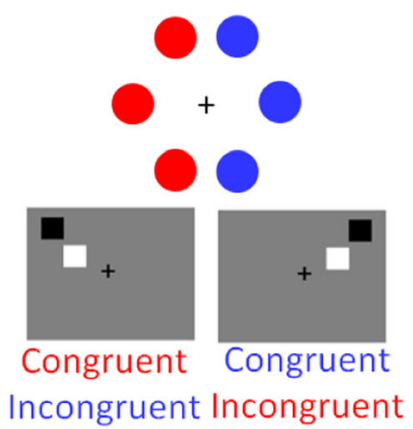

within each hemifield. After an initial delay (Delay 1), color distractors representing the previously rewarded (e.g., red), or novel (e.g., blue) colors were displayed. Following a second delay period (Delay 2), the test array was displayed. Participants had to determine whether the two squares (a) straddled the imaginary line (Location trials), or (b) had the same vertical relationship (Relation trials). B, Position of contralateral electrodes relative to the previously rewarded (e.g., red) or novel (e.g., blue) distractor. C, Congruency between the hemifield of the distractors and the WM test items

is the white square above or below the black square?). Upon test, participants indicated whether or not the squares in the test array had the same relative positions as the sample squares in that hemifield. For both trial types, participants pressed one button for a "match" response and another for a "non-match" response, and these response key mappings were counterbalanced between participants. Trial types were pseudo-randomly presented so participants could not predict what trial type they would see until the cue. While EEG was recorded, participants completed a total of 540 trials (270 Location, 270 Relation randomly intermixed), which were broken down into nine runs of 60 trials per run. Half of the WM test trials had the test array in the left hemifield. Moreover, half of the WM test trials had the test array in the same hemifield as the distractor's hemifield. The additional two training sessions with the visual search task for reinforcing the learning of the vertical positions of the two squares in each hemifield (i.e., 
reward-color association were interspersed between runs 3 and 4 and between runs 6 and 7 .

EEG recording and preprocessing In an electromagnetically shielded room, EEG data were recorded at 32 sites covering the whole scalp with approximately uniform density using an electrode cap referenced to the $\mathrm{Cz}$ electrode during recording (ActiCHamp, Brain Products, Munich, Germany). Electrode impedance was kept below $20 \mathrm{k} \Omega$. All EEG electrodes were recorded continuously in DC mode at a sampling rate of 512 Hz. EEG data were processed with Fieldtrip (Oostenveld et al., 2011). Data were first corrected for difference in timing between the stimulus program triggers and the photodiode activity, and then were filtered $(0.3-30 \mathrm{~Hz}$ bandpass for ERPs and $0.5-100 \mathrm{~Hz}$ for time-frequency analyses), and baselinecorrected (i.e., first $200 \mathrm{~ms}$ of the fixation period). This baseline was selected in line with previous work (e.g., Ikkai et al., 2014) and to avoid any time period that might include WM processes. For ERP analyses, data were segmented into epochs covering the time from $3.4 \mathrm{~s}$ before to $3.3 \mathrm{~s}$ after the onset of distractors, while for time-frequency analyses the epochs covered the $6.7 \mathrm{~s}$ of the trial, locked to the onset of fixation. Independent components analysis (ICA) was performed on the epoched data, and the eye-blink component was identified and removed for each participant's data. Trials containing horizontal eye movements were rejected entirely (mean percentage of rejected trials: $17 \%$, range: 9-29\%). Artifact-free epochs for each experimental sequence were averaged separately to obtain ERPs in each participant. Both previously rewarded and non-rewarded distractors were investigated while the novel color was displayed in the other hemifield (Fig. 2B). Power spectra were calculated using a time-frequency transformation based on multiplication in the frequency domain from 1 to $30 \mathrm{~Hz}$ with $0.5-\mathrm{Hz}$ increments using a hanning taper applied to short sliding time windows (Percival \& Walden, 1993) every 100 ms. An adaptive time window of five cycles for each frequency $(\Delta \mathrm{T}=5 / f)$ was applied. Time-frequency was computed at the single-trial level before averaging, thus showing induced (i.e., non-phaselocked) activity.

Statistical analyses Error trials were excluded from further analysis. For behavioral analyses, we tested for differences in mean accuracy and response time (RT) as a function of the display of the previously rewarded, or non-rewarded color distractor, and as a function of the congruency between the hemifield of the rewarded/non-rewarded distractor and of the WM test array, for both Relation and Location trials. We used 2 (Trial type: Location, Relation trials) $\times 2$ (Distractor: previously rewarded, previously non-rewarded) $\times 2$ (Congruency: congruent hemifield between the display of the rewarded/nonrewarded distractor and of the WM test array, incongruent hemifield) repeated-measures ANOVAs. Unless otherwise noted, only effects significant to at least $\mathrm{p}<0.05$ were reported.
To correct for multiple comparisons, Sidak correction was applied (e.g., Abdi, 2007), and Sidak-adjusted p-values were reported.

Statistics and visualization of EEG data were done using Brainstorm software (Tadel et al., 2011). For ERP and timefrequency analyses, we used nonparametric permutation tests (Maris \& Oostenveld, 2007) to statistically test for differences between Relation and Location trials. First, the selected electrodes of interest $(\mathrm{O} 2, \mathrm{P} 4, \mathrm{P} 8, \mathrm{CP} 6$; and $\mathrm{O} 1, \mathrm{P} 3, \mathrm{P} 5, \mathrm{CP} 5)$ were grouped in a cluster. Activations within this cluster were distinguished on the basis of whether they were contralateral or ipsilateral to the previously rewarded, non-rewarded, or novel color. This electrode cluster was selected a priori based on previous work on WM and attentional capture (e.g., Blacker et al., 2016; MacLean \& Geisbrecht, 2015; Qi et al., 2013). Our time range of interest was the onset of the distractor and the following Delay 2 period. This period was selected to specifically investigate how the processing of the distractors influenced the WM maintenance period. No differences were expected during the WM test phase because of the influence of motor response and the jittered timing relative to the onset of the test cue stimulus. For every time point within the selected cluster, differences in activation contralateral to rewarded/nonrewarded, and novel distractors were tested for significance using permutation across conditions $(n=1,000)$, with FDR (i.e., False Discovery Rate; Benjamini \& Hochberg, 1995) correction for multiple comparisons. This function first performed a $t$-test at each time point. For each iteration randomizing trial labels, clusters of electrodes where the alpha-level was $<0.05$ were identified, and their $t$-values were summed. The largest sum of $t$-values was used as a $t$-statistic. This procedure was repeated to create the null distribution. The p-value was estimated according to the proportion of the null distributions exceeding the observed cluster-level $t$-statistic. Correlations were FDR corrected for multiple comparisons.

\section{Results}

Reward training task For RT (Fig. 1b), faster responses were observed across training blocks $(646 \mathrm{~ms}, 631 \mathrm{~ms}$, and $626 \mathrm{~ms}$, respectively), $F(2,36)=5.29, p<0.01, M S \mathrm{e}=230.03, \eta p^{2}=0.23$, and for rewarded compared to non-rewarded colors $(608 \mathrm{~ms}$ and $661 \mathrm{~ms}$, respectively), $F(1,18)=27.34, p<0.001$, $M S \mathrm{e}=4513.31, \eta p^{2}=0.60$. There was also a significant Block $\times$ Reward interaction, $F(2,36)=3.28, p<0.05, M S e=54.70$, $\eta p^{2}=0.15$, as the difference between rewarded and nonrewarded colors was larger during the third block $(593 \mathrm{~ms}$ and $658 \mathrm{~ms}$, respectively) than during the previous blocks (block 1: $622 \mathrm{~ms}$ and $670 \mathrm{~ms}$; block 2: $608 \mathrm{~ms}$ and $654 \mathrm{~ms}$, respectively). Analyses on accuracy revealed higher performance when the rewarded color was displayed compared to the non-rewarded color (91.0\% and $81.0 \%$, respectively), 
$F(1,18)=10.96, p<0.005, M S e=161.59, \eta p^{2}=0.38$. The Block $\times$ Reward interaction was also significant, $F(2,36)=4.23$, $p<0.03, M S e=8.71, \eta p^{2}=0.19$, with a larger difference between rewarded and non-rewarded colors during the last block (92.3\% and $78.2 \%$, respectively) compared with the previous blocks (block 1: $88.1 \%$ and $82.1 \%$; block 2: $93.0 \%$ and $82.6 \%$, respectively).

WM task: Behavioral data For RT (Fig. 3), faster responses were observed for Location $(689 \mathrm{~ms})$ than for Relation trials $(751 \mathrm{~ms})$, $F(1,18)=41.52, p<0.001, M S e=8.21, \eta p^{2}=0.70$. There was a significant Trial type $\times$ Distractor $\times$ Congruency interaction, $F(1,18)=16.39, p<0.001, M S \mathrm{e}=248.95, \eta p^{2}=0.48$. The Distractor $\times$ Congruency interaction was significant for Relation trials, $F(1,18)=16.57, p<0.001, M S e=214.06$, $\eta p^{2}=0.48$. Planned comparisons revealed that participants were faster to respond to the WM test array when the hemifield was congruent with the display of the previously rewarded distractor than when it was incongruent (740 ms vs. $757 \mathrm{~ms})$, $F(1,18)=5.89, p<0.026, M S e=7.08, \eta p^{2}=0.25$. Moreover, participants were faster to respond to WM targets that were congruent to a previously rewarded distractor than congruent to a previously non-rewarded distractor (740 ms vs. $760 \mathrm{~ms}$ ), $F(1,18)=17.65, p<0.001, M S e=4.92, \eta p^{2}=0.50$. There was also a significant Trial type $\times$ Distractor interaction, $F(1,18)=6.07$, $p<0.024, M S e=72.79, \eta p^{2}=0.25$, as the difference between rewarded and non-rewarded distractors was significant for Relation trials (rewarded distractor minus non-rewarded distractors: $29 \mathrm{~ms}), F(1,18)=19.07, p<0.001, M S \mathrm{e}=5.49$, $\eta p^{2}=0.51$, and non-significant for Location trials (-6 ms), $F<1.1$.

For accuracy, no difference was observed between Location and Relation trials $(F<2.2)$. However, the Trial type $\times$ Distractor $\times$ Congruency interaction was significant, $F(1,18)=20.43, p<0.001, M S \mathrm{e}=18.78, \eta p^{2}=0.53$. The Distractor $\times$ Congruency interaction was significant for Relation trials, $F(1,18)=22.11, p<0.001, M S \mathrm{e}=33.27$, $\eta p^{2}=0.55$, and non-significant for Location trials, $F<1.0$. On Relation trials, accuracy was significantly higher when the WM test hemifield was congruent with the previously rewarded distractor hemifield than when it was incongruent $(91.0 \%$ vs. $84.7 \%), F(1,18)=13.96, p<0.002, M S e=1.19$, $\eta p^{2}=0.44$. Conversely, following the display of the nonrewarded distractor, accuracy was significantly higher when the WM test hemifield was incongruent than congruent to the distractor hemifield $(91.5 \%$ vs. $86.5 \%), F(1,18)=19.56$, $p<0.001, M S e=1.53, \eta p^{2}=0.52$. The Distractor $\times$ Congruency interaction, $F(1,18)=8.24, p<0.01, M S e=14.62, \eta p^{2}=0.31$, revealed that the effect of the congruency between the distractor and WM test hemifield was significant when the distractor was the previously rewarded color, $F(1,18)=5.13, p<0.036$, $M S e=1.34, \eta p^{2}=0.22$, but non-significant when the previously non-rewarded color was displayed, $F<4.0$. Thus, accuracy and RT data are consistent in demonstrating better performance in the Relation WM task when WM is tested in the same hemifield as where the distractors (that were the
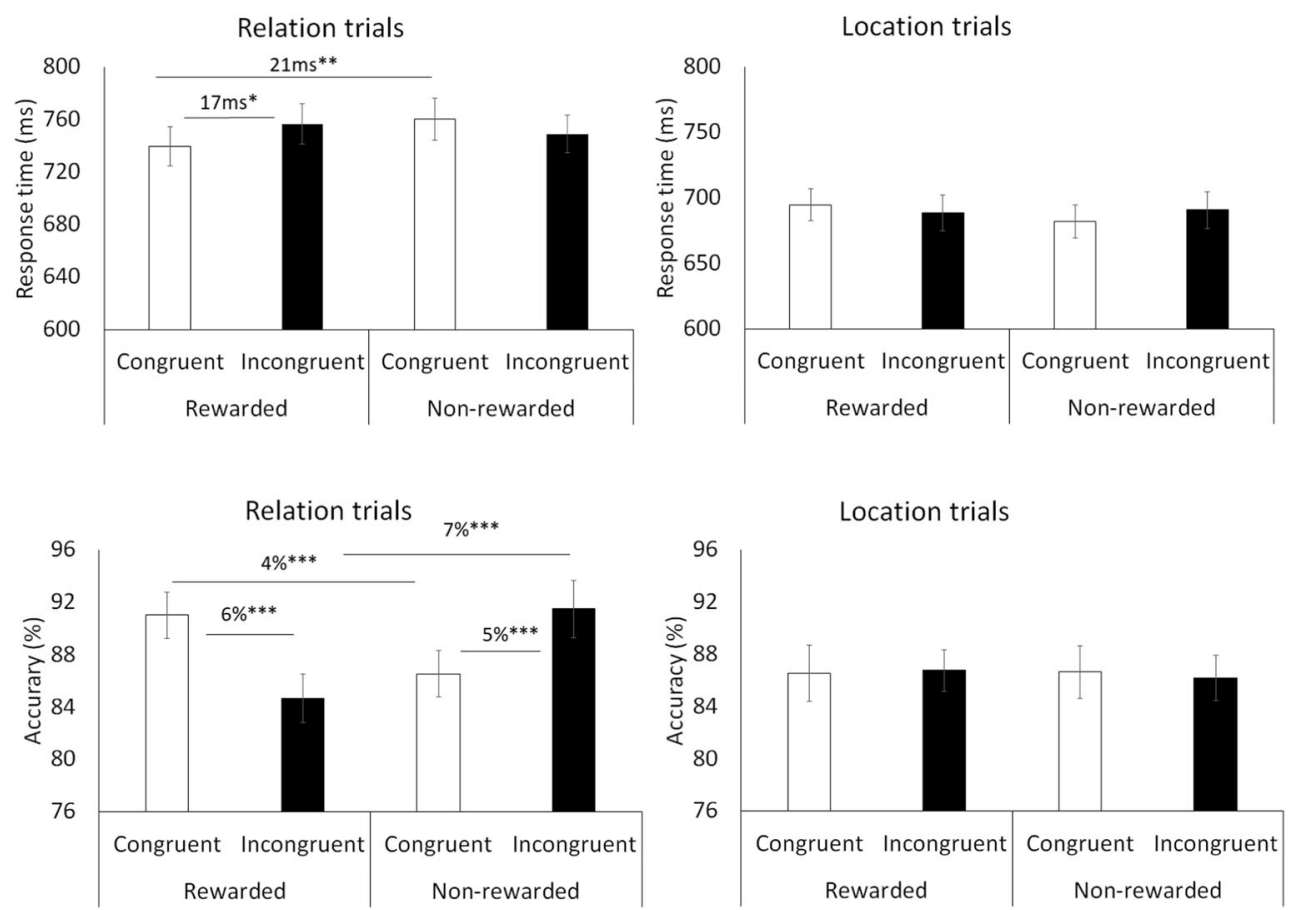

Fig. 3 Behavioral performance shown separately for Location and Relation trials. Performance was modulated as a function of the display of a previously rewarded or non-rewarded distractor, and as a function of

the congruency between the hemifield of the distractor and the WM test items. Errors bars represent S.E.M. ${ }^{*} \mathrm{p}<0.5,{ }^{*} \mathrm{p}<0.01, * * * \mathrm{p}<0.001$ 

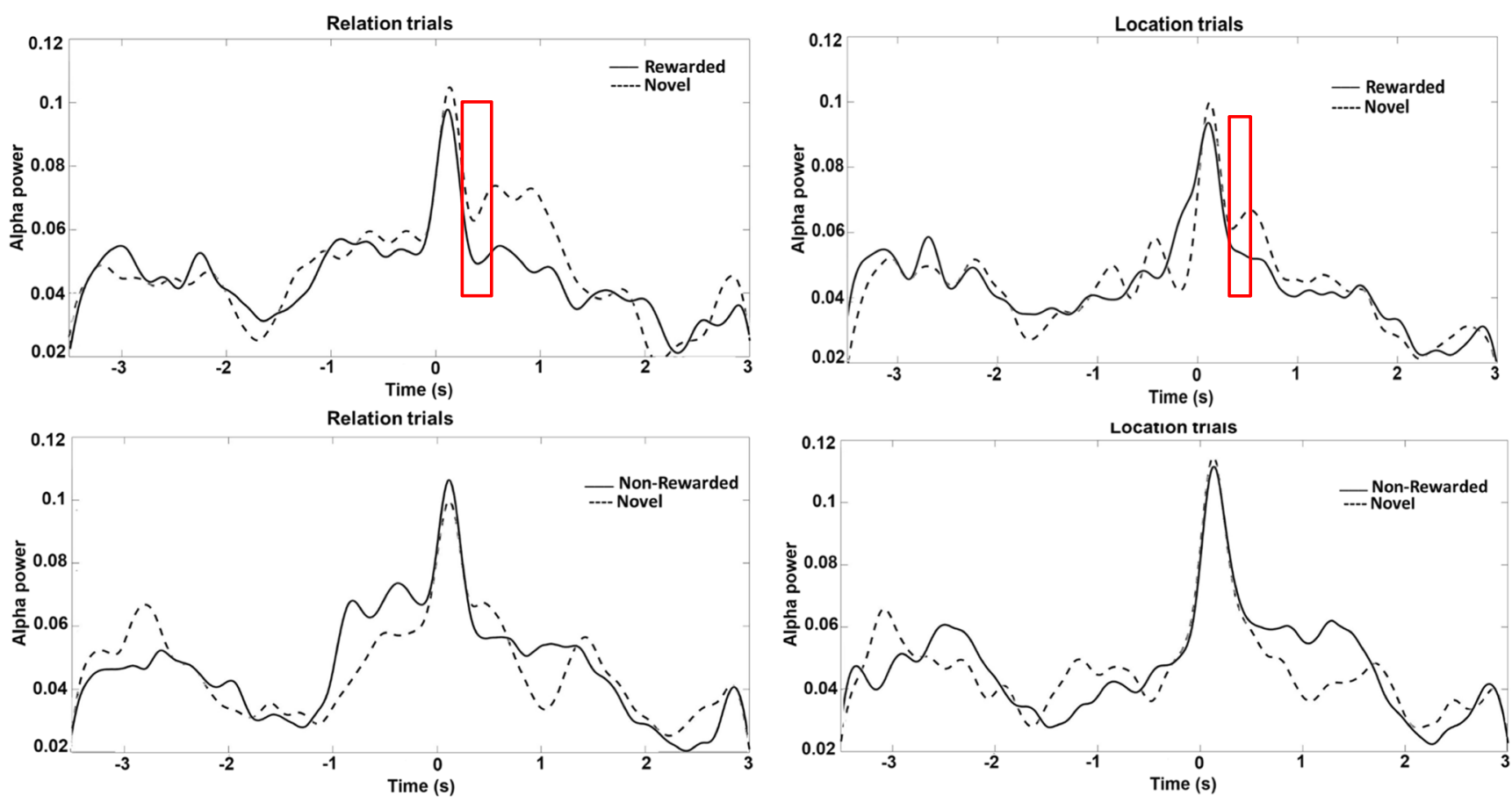

Fig. 4 Time-frequency results for posterior electrodes, for relation (left) and location (right) trials. Alpha power for electrodes contralateral to the previously rewarded or novel distractor. Zero represents the onset of the

same color as the previously rewarded target stimuli) appeared.

Time-frequency analyses of EEG data We contrasted alpha power for Relation and Location trials as a function of whether a previously rewarded, non-rewarded, or novel distractor was displayed contralateral to the electrodes of interest (Figs. 4 and

distractor. The red boxes represent the significant differences between conditions revealed by permutation tests in relation trials $(\mathrm{p}<.01)$ and location trials $(\mathrm{p}<.05)$

5). Permutation tests revealed that, for Relation trials, during the memory delay following the distractor stimuli, alpha power was significantly lower on the electrode cluster contralateral to the previously rewarded color (0.054), compared to the same electrode cluster contralateral to the novel color $(0.071$, $p \mathrm{~s}<0.01$ ). Results were observed between $192 \mathrm{~ms}$ and $348 \mathrm{~ms}$ after the onset of the distractor. For Location trials, between

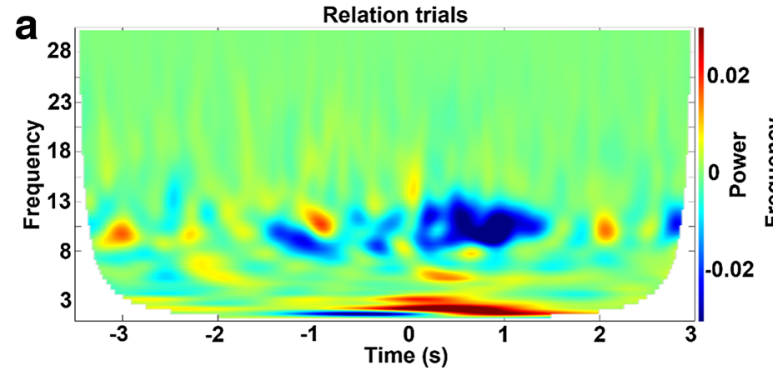

b

Relation trials
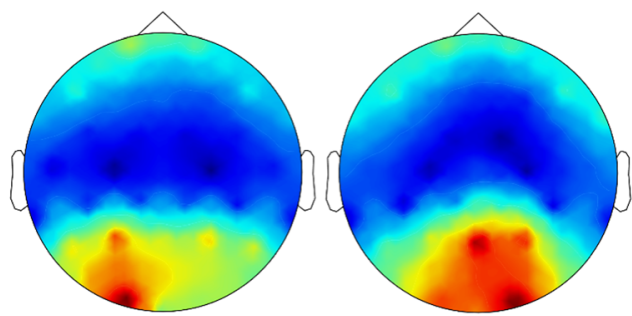

Fig. 5 A, Time-frequency maps of the previously rewarded distractor minus novel distractor difference, at posterior sites, for relation and location trials. B, Scalp map of power in the alpha band $(8-13 \mathrm{~Hz})$, during the

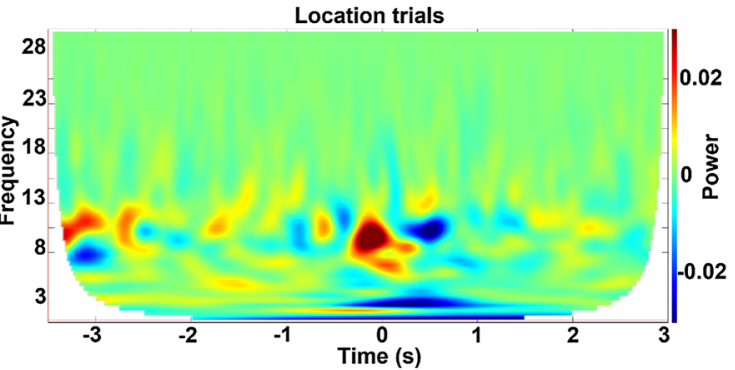

Location trials
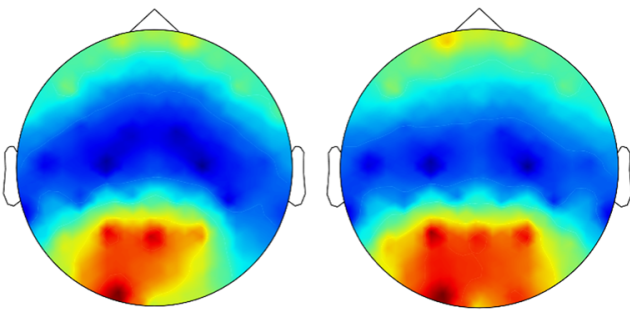

significant time cluster for relation trials (192-348ms), when the previously rewarded distractor was displayed in the right hemifield (left, contralateral sites) or the left hemifield (right) 
$278 \mathrm{~ms}$ and $316 \mathrm{~ms}$, alpha power for the electrode cluster contralateral to the previously rewarded color $(0.62)$ was found to be lower than that contralateral to the novel color $(0.56, p s<0.05)$. No significant modulations were observed for the non-rewarded color relative to the novel color, or preceding/following the distractor phase. Because the rewarded and non-rewarded distractors were always paired with a novel distractor and not with each other, however, alpha power could not be directly contrasted between rewarded and non-rewarded distractors. To control for the influence of the familiarity difference between the previously rewarded and non-rewarded distractors versus the novel distractor, we also calculated an index of the influence of the previously rewarded distractor for both Location and Relation trials: (Contralateral to previously rewarded distractor minus Contralateral to novel distractor) minus (Contralateral to previously non-rewarded distractor minus Contralateral to novel distractor). Results revealed a significantly lower alpha power

a
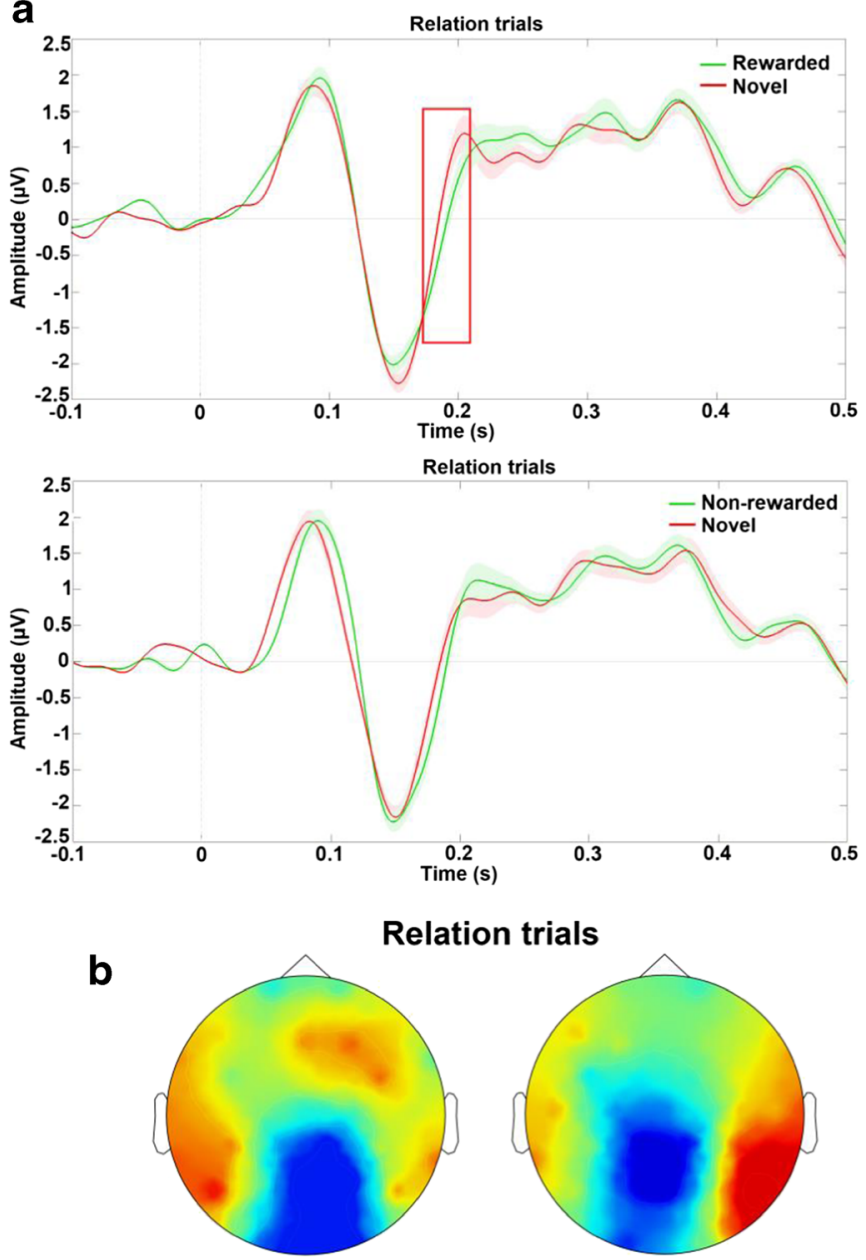

Relation trials

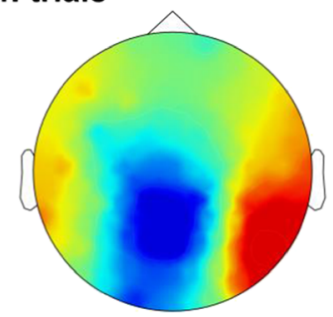

Fig. 6 A, Event-related potentials (ERPs) for posterior sites as a function of the trial type (relation trial, location trial) and the electrode position contralateral to the hemifield of the rewarded, non-rewarded, or novel distractor. Zero represents the onset of the distractor. The red box represents the significant difference between conditions revealed by in the same latencies for rewarded relative to non-rewarded distractors during Relation trials $(p s<0.01)$. An additional ANOVA was conducted on the mean activations within the significant cluster. Results revealed a marginally significant Trial type $\times$ Distractor interaction, $F(1,18)=4.23, p<0.055$, $M S \mathrm{e}=0.02, \eta p^{2}=0.19$, with a larger difference between rewarded and novel trials for Relation trials than for Location trials.

Event-related potentials We contrasted ERPs of Relation and Location trials as a function of whether a previously rewarded, non-rewarded, or novel distractor was displayed contralateral to the electrodes of interest (Fig. 6). Permutation tests revealed that amplitudes at contralateral sites were more negative for previously rewarded distractors $(M=0.17 \mu \mathrm{V})$ than for novel distractors $(M=0.67 \mu \mathrm{V})$, in line with the $\mathrm{N} 2 \mathrm{pc}$ component. This difference was observed between $180 \mathrm{~ms}$ and $210 \mathrm{~ms}$ after onset of the distractor $(p<0.005)$, and was observed for
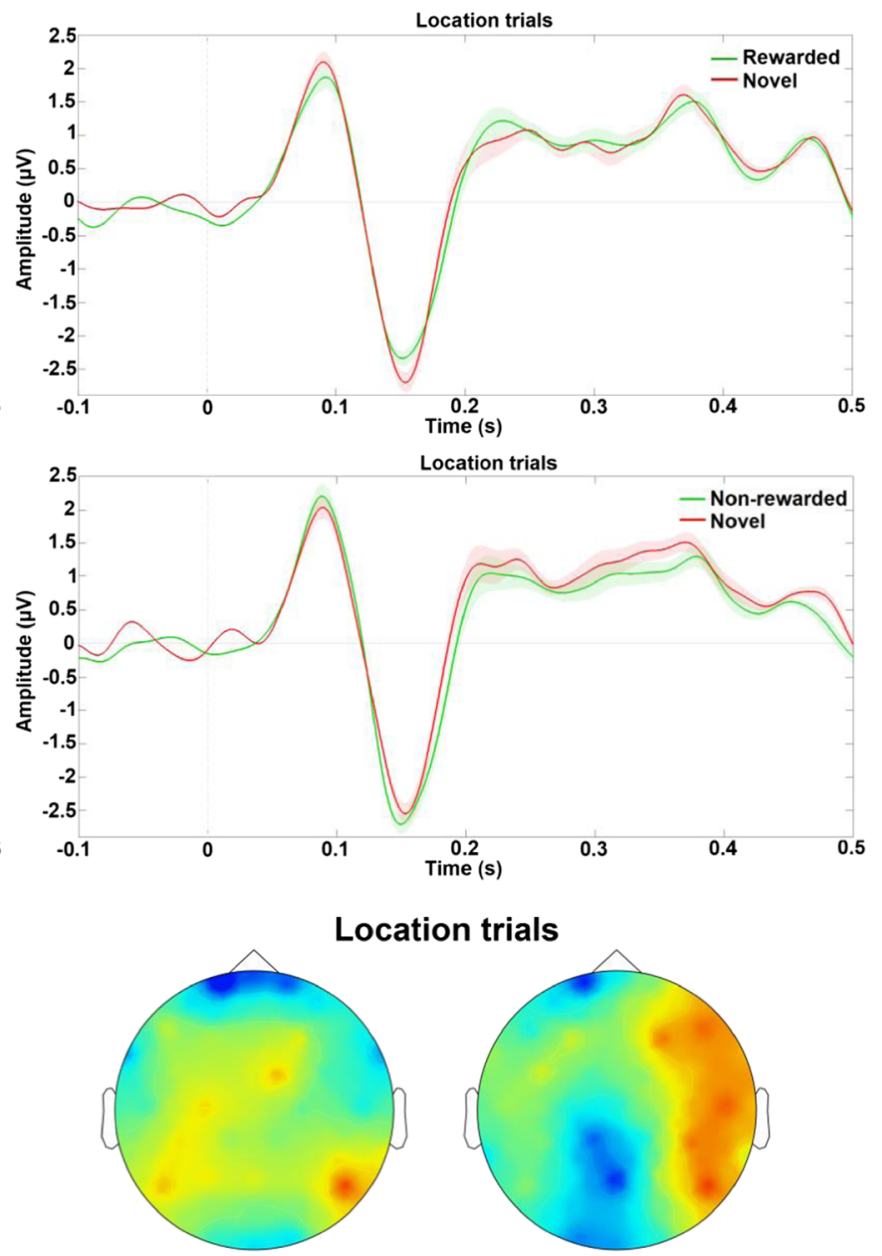

permutation tests $(\mathrm{p}<.005)$. B, Scalp map of activation during the significant time cluster for Relation trials (180-210 ms) when the previously rewarded distractor was displayed in the right hemifield (left, contralateral sites) or the left hemifield (right) 
Relation trials only, as no significant modulations were observed for Location trials. In addition, no significant modulations were observed for non-rewarded distractors in either Location or Relation trials. Analyses with the same rewarded versus non-rewarded index as was used for the alpha power analysis revealed similar results $(p<0.001)$, which indicated more negative $\mathrm{N} 2 \mathrm{pc}$ for rewarded than for non-rewarded distractors. An additional ANOVA was conducted on the mean activations within the significant cluster. Results revealed a significant Trial type $\times$ Distractor interaction, $F(1,18)=7.49, p<0.02, M S \mathrm{e}=0.10, \eta p^{2}=0.29$. The difference between rewarded and novel trials was larger for Relation trials $(-0.50 \mu \mathrm{V})$ than for Location trials $(-0.18 \mu \mathrm{V})$.

Furthermore, correlations were conducted between behavioral modulations and the significant ERP/time-frequency clusters. Correlations with training data revealed a negative correlation between the significant N2pc amplitude difference for previously rewarded distractors during Relation trials and the change in the rewarded minus non-rewarded RT difference from the first to the last session of the training phase ( $r=-$ $0.606, p<0.002$; Fig. 7). This correlation indicates that the $\mathrm{N} 2 \mathrm{pc}$ component, indicating reward-related distraction, was
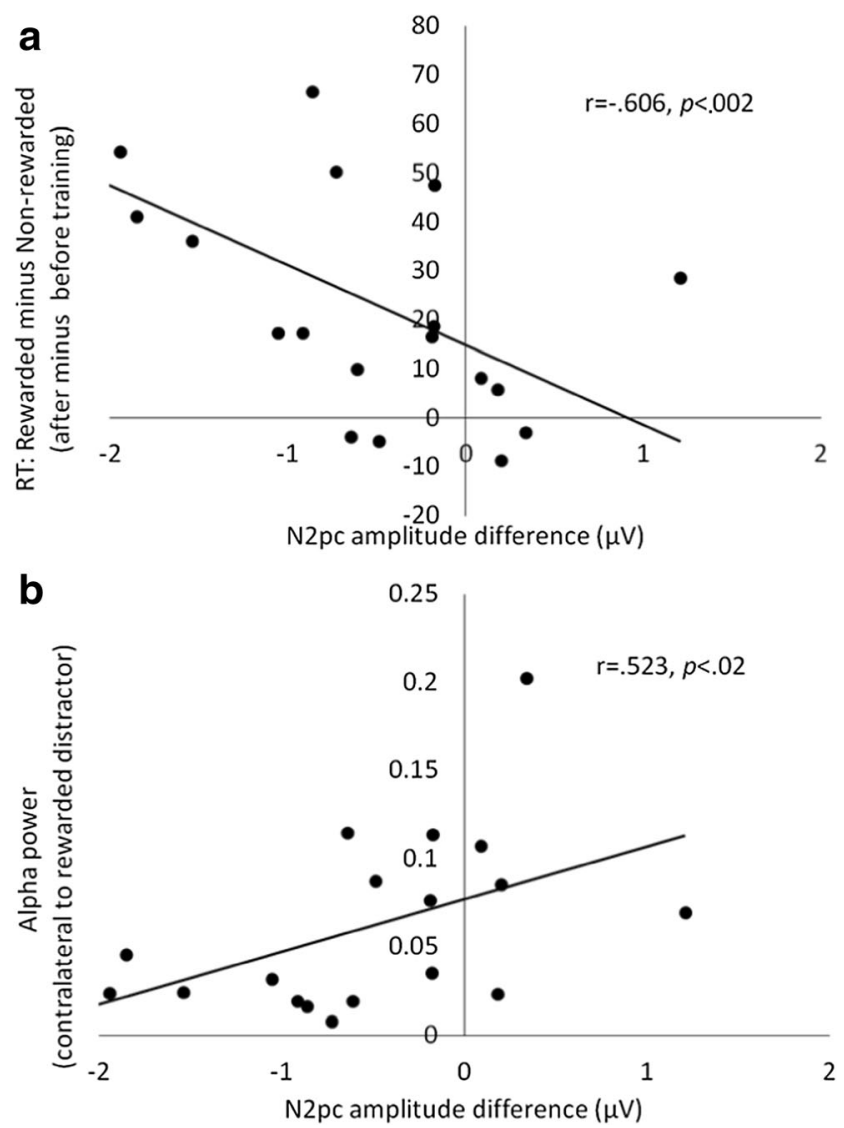

Fig. 7 A, correlation between the change in the rewarded minus nonrewarded RT difference from the first to the last session of the training phase and N2pc cluster revealed by permutation tests. B, correlation between N2pc amplitude and alpha power contralateral to the previously rewarded distractor more negative in individuals who showed larger reward learning across blocks during the training phase. Moreover, correlations revealed that the significant modulations of alpha power for rewarded distractors in Relation trials (between $200 \mathrm{~ms}$ and $250 \mathrm{~ms}$ following distractor onset) were positively correlated to the N2pc amplitude difference $(r=0.523, p<0.022)$, suggesting that a more negative N2pc was also associated with a larger reduction of alpha power.

\section{Discussion}

In the present study, we used both ERP and time-frequency analyses to test whether neural correlates of VDAC differed as a function of the type of information maintained in WM. Behavioral measures of reward-related attentional bias were obtained during training blocks before and between WM task runs. During the WM task, although RTs were overall faster in Location trials relative to Relation trials (see also Ikkai et al., 2014), both trial types were similar in terms of accuracy, which is not consistent with a difference of difficulty between trial types regarding WM maintenance. Therefore, the RT difference likely reflects extra computations required during the test phase of Relation trials, to convert the sensory test stimulus input to a relational representation that can be compared to the relational information from the sample stimulus that was presumably maintained during the delay of those trials, rather than differences in maintenance difficulty or reward-related processing. In addition to previous work (Blacker et al., 2016; Blacker \& Courtney, 2016; Ikkai et al., 2014), the present results provide converging evidence for differential reliance on sensory information between Relation and Location trials that is independent of difficulty or memory load. In line with previous work (e.g., Blacker et al., 2016; Ikkai et al., 2014; Qi et al., 2013), we studied the modulation of EEG alpha power and the N2pc component over posterior electrodes, and we contrasted activations that were contralateral to a previously rewarded, non-rewarded, or novel color distractor. Results revealed that, following the distractor display, alpha power was lower over posterior electrode sites contralateral to the previously rewarded color. In addition, we observed an N2pc component with more negative potential contralateral to the distractor with the previously rewarded color. These effects were observed during relational WM, and were reduced (i.e., alpha power) or absent (i.e., N2pc) during location WM maintenance. Furthermore, they were found only for the previously rewarded color, not for distractors whose color was not associated with reward. In both cases, a novel color was displayed in the other hemifield. Although some uncertainty remains regarding how relation information is maintained, these results likely reflect the larger interference of previously rewarded sensory information during maintenance of abstract relational than sensory location information, and 
suggest that VDAC differs as a function of the type of information maintained in WM or differentially impacts these processes.

In addition to these neural activity results, behavioral results for the training and WM tasks are also consistent with the interpretation that these distractor stimuli that were currently irrelevant resulted in what has been termed VDAC (Anderson et al., 2011; Rutherford et al., 2010). During the training task, the difference between rewarded and non-rewarded stimuli increased across blocks, consistent with reward learning. As with the neural data, the behavioral results during the WM taskalso revealeda modulation of this capture by the nature of the information maintained in WM. For Relation trials only, participants were faster and more accurate when the hemifield of the WM test matched the hemifield of the previously rewarded distractor, compared to the other hemifield. This result was interpreted as reflecting the influence of attentional capture, and suggests a facilitating effect when the distractor and the subsequent WM test are displayed in the samehemifield. Conversely, decreased performance was observed when the hemifields of the distractor and of the WM tests were different. The shift of attention away from the incongruent hemifield might facilitate WM performance within the congruent hemifield by reducing the strength of the representations of items in the incongruent hemifield and thus reducing potential response conflicts at the time of the WM behavioral test within the congruent hemifield. Performance was also significantly worse when the WM test hemifield matched a previously nonrewarded distractor, compared to the other hemifield, which contained a novel color. Results may be interpreted as a bias to orient to novel, infrequent stimuli, over familiar stimuli (e.g., Anderson et al., 2012; Johnston et al., 1990; Johnston \& Schwarting, 1997; Neo \& Chua, 2006), as the non-rewarded distractor had a higher familiarity than the novel distractor without being associated with value. The results suggestan attentional priority hierarchy, without physical salience or task relevance differences, in which the previously rewarded stimulus has the highest priority, a familiar but not rewarded stimulus has the lowest priority or is suppressed, and a novel stimulus has a priority between these two. Regarding suppression, however, previous work showed thatnegativestimuli tend to receiveattentional priority rather than being suppressed (e.g., Failing \& Theeuwes, 2018). No behavioral evidence of differential attentional capture according to reward or familiarity was observed for Location trials. If spatial attention was allocated to specific retinotopic locations to maintain the position of the imaginary lines during Location trials, this focused selectiveattention could haveresulted in sufficient filtering of the value-associated distractor so as to reduce capture of attention, resulting in no measurable effect of VDAC on Location WM performance. Conversely, Relation WM does not benefit from retinotopically focused attention and thus might leave visual attention more susceptible to capture by the previously rewarded distractor, leading to greater influences on the WM task performance. As previous work showed that attention can be captured by previously rewarded stimuli even when attention is spatially focused (e.g., Munneke et al., 2016), the lack of an effect in the current study might reflect specific WM-related processes.

Regarding EEG results, alpha power was previously associated with allocation of attention processing and the selective inhibition of posterior areas (e.g., Hinault et al., 2016; Ikkai et al., 2014; Van Diepen et al., 2016). In the present study, following the display of the distractor, alpha power was lower on the cluster contralateral to the previously rewarded distractor, compared to when a novel distractor was displayed. The observed effects are thus in line with attentional capture and greater visual processing of the reward-associated distractor (see also Harris et al., 2017). Given that these modulations were only observed following the rewarded distractor cue, results cannot be accounted for as reflecting WM maintenance processes. This modulation was observed on both Relation and Location trials, but the comparison of the difference between rewarded and non-rewarded distractors as a function of trial types showed that effects were larger in Relation than in Location trials. This result suggests that, even if no behavioral effects were observed in Location trials, the value-associated distractor may have led to attentional bias in both trial types. The reduced alpha modulations for Location trials is consistent with the distractor information being processed but being filtered and/or not resulting in attentional reallocation, so that behavioral performance was not influenced. In contrast with previous work (e.g., Blacker et al., 2016), no frontal modulations of alpha band activity were observed in the present study. In the current task, the trial type was indicated at the beginning of the trial, not following the memory sample array. Therefore, WM updating and representation conversion processes, which have been shown to engage frontal brain regions (see Nee et al., 2013, for a meta-analysis) were not engaged during the Delay 2 period. In line with previous works (e.g., Ikkai et al., 2014), no significant modulation was observed during the test period.

Moreover, ERP analyses revealed modulations of the N2pc components during the Delay 2 period, with larger negativity on posterior sites contralateral to the previously rewarded distractor than those contralateral to the novel distractor. In line with previous work (e.g., Kumar et al., 2009, 2016), this effect might also extend to the N1 component. However, the similarity of the P1-N1 complex across conditions suggests that the $\mathrm{N} 2 \mathrm{pc}$ for the previously-rewarded cue in the Relation trial is driving the effect. Consistent with the observed alpha modulations, this effect suggests attentional capture by the previously rewarded distractor, as similar N2pc effects were previously observed for value-associated distractors (e.g., Itthipuripat et al., 2015; Qi et al., 2013). However, this effect was only observed in Relation trials, suggesting that attentional capture was larger during Relation trials than during Location trials, in line with behavioral results. Previous work also showed VDAC on the P1 component (e.g., MacLean \& Giesbrecht, 2015), 
which was not significantly modulated in the present study. We can hypothesize that reward history influences target selection processes rather than early spatial selection processes.

The N2pc amplitude difference for rewarded versus novel distractors during Relation trials was significantly correlated with induced alpha power contralateral to the previously rewarded distractor. The N2pc amplitude difference was also correlated with the augmentation of the rewarded minus nonrewarded reaction time difference from the first to the last training period. Results suggest that participants with the largest learned association between the color of the distractor and the previous reward also showed the largest N2pc amplitude to the previously rewarded distractor. The correlation between $\mathrm{N} 2 \mathrm{pc}$ and alpha power is also interesting, and is in line with previous work showing an association between oscillatory activity and event-related responses (e.g., Sokhadze et al., 2009; van Dijk et al., 2010). Importantly, this correlation was observed with non-phase-locked activity, suggesting that the correlation is not only reflecting event-related effects. Results suggest that investigating cognitive processing with both ERPs and time-frequency analyses provide complementary findings to better understand the cognitive processes involved (see also Hinault \& Lemaire, 2017). All in all, results suggest that the influence of VDAC on WM maintenance is stronger during relation WM than during location WM.

The present study raises important points regarding how the cognitive processes differ between relational and sensory WM. However, the specification of the processes involved is still a matter of investigation, and several alternative hypotheses to the present interpretation need to be addressed. Indeed, the differential reliance on sensory strategies (see Hinault \& Lemaire, 2016, for an overview) between Location and Relation trials remains to be further specified. During the training task in which the reward association was established, an RT difference emerged for the rewarded versus non-rewarded stimuli, indicating the development of reward-related attentional bias that would be expected to result in VDAC as shown in previous research using search tasks to behaviorally test for the existence of VDAC. The effects of these previously rewarded stimuli as completely irrelevant distractors during our WM tasks, however, may have had a different effect than that observed during the training phase or during other VDAC research test phase tasks (e.g., Anderson, 2015). Behavioral and EEG effects of previously rewarded information were observed in the WM task, but it is somewhat unclear how much of the interaction between the type of distractor (rewarded/non-rewarded/novel) and the type of WM task (Relation/Location) was due to reward and how much to other attentional biases and processes that may have differed during the WM task delays. One alternative interpretation to the results would be that attention might have been captured and then suppressed/disengaged by the time the Location test appeared. In addition to the lack of behavioral results, the reduction of reward-related EEG effects for Location trials compared to Relation trials suggests that differences mainly occurred during the distractor encoding stage rather than during the processing of the test array and response preparation. Moreover, rewarded ( $\$ 0.25$ on $80 \%$ of correct responses trials and $\$ 0.10$ on $20 \%$ of correct responses) and non-rewarded (always followed by $\$ 0.00$ ) colors differed in certainty of the outcome during training, and results could be interpreted as reflecting uncertainty associations rather than reward. However, participants showed larger capture to rewarded stimuli even though their predictive value was lower, suggesting that attention bias toward stimuli with more certain outcomes (e.g., Marchner \& Preuschhof, 2018) was not driving the results. Other studies, however, have reported attention bias towards less certain stimuli (e.g., Le Pelley et al., 2018), but even in that study reward association influenced attention bias to a greater degree than uncertainty. In addition, previous work showed attentional capture by previously rewarded stimuli while controlling for certainty (e.g., Qi et al., 2013) between rewarded and non-rewarded conditions. Previous research has indicated that attention bias for stimuli with uncertain outcomes depends on whether further learning is required (e.g., Maddux et al., 2007), which was not the case in the current study. Taken together, these results suggest that reward, rather than certainty, is driving the current reported effects. A final possibility that cannot be ruled out by the present results is that item encoding during Relation trials, and the maintenance of luminance relationships as well as spatial relationships, could have resulted in less color filtering during the distractor phase, as the luminance (i.e., color) dimension of the remembered information is not completely irrelevant in the Relation trials as it is in the Location trials. Future studies will aim at investigating the conditions of occurrence of VDAC, and the influence of visual WM capacity. Reduced visual WM capacity could be related to increased attentional capture during Location trials, as the focused attention of specific location would be less efficient than in individuals with high visual WM capacity. Future studies (including eye-tracking) will aim to further our understanding of the cognitive processes involved during Relational and Location WM.

Recent work showed that attentional capture was rewardbased but was also influenced by the familiarity of previous targets (e.g., Sha \& Jiang., 2016, but see Anderson \& Halpern, 2017). In the present study, modulations of both alpha power and N2pc amplitude were found following the previously rewarded distractor, compared to a novel color. The difference between responses to rewarded versus non-rewarded distractors, correcting for the simultaneous presence of the novel distractor in the opposite hemifield, was significantly greater during relation WM than during location WM, suggesting that the present results reflect VDAC rather than purely familiarity.

The presented results have several theoretical implications, as they contribute to a better understanding of the influence of VDAC on WM maintenance, and how this influence is modulated by the type of information maintained in WM. The current results have implications for current frameworks of WM 
processing (e.g., Baddeley et al., 2010; Barrouillet et al., 2004; Barrouillet and Camos, 2010; Courtney, 2004; Vandierendonck, 2016), as they provide evidence that (a) the nature of attentional capture by distracting stimuli can modulate the efficacy of WM maintenance, and (b) the relationship between WM and attention depends on the type of information maintained in WM. Allocation of attention is sometimes considered as a part of these models, but these models mostly consider the maintenance of sensory information. Evidence, such as that provided in the current study, of modulations of selective attentional capture or differential influence according to whether sensory versus abstract information is maintained in WM needs to be considered when trying to understand the cognitive and neural mechanisms that enable WM. The present study contributes to this issue by specifying the time course of attentional capture during WM maintenance, and what WM processes are the most influenced by attentional capture. Indeed, results revealed that that abstract (relational) WM representations are more vulnerable to nonrelevant yet previously rewarded stimuli than are sensory representations, during early processing stages. Conversely, sensory representations that involve allocation to a specific location might be less subject to attentional capture by irrelevant information. Distracting stimuli have previously been shown to have different magnitudes of impact on sensory WM performance depending on whether the information domains of the maintained information and the distracting information overlap or not (e.g. Baddeley, 2007). The current study extends this general approach to investigate the potential for similar interactions between processing versus maintenance in very different information domains than have been traditionally studied. In order to maximize power to detect a difference in the distraction effects of previously rewarded versus non-rewarded distractors, we did not have any trials that contained no distractors, which would be needed to investigate whether relational WM is more susceptible to sensory distraction in general. So, while the current study demonstrated that previously rewarded stimuli have greater impact on relational WM, future studies will be needed to further investigate the generality of the interaction between attention capture and WM content, how it might be further modulated by training, or might differ between populations.

Acknowledgements This work was supported by a Johns Hopkins Science of Learning Institute Fellowship to KJB, and by NIH grant RO1-DA013165 to S.M.C.

Publisher's Note Springer Nature remains neutral with regard to jurisdictional claims in published maps and institutional affiliations.

\section{References}

Abdi, H. (2007). Bonferroni and Šidák corrections for multiple comparisons. Encyclopedia of Measurement and Statistics, 3, 103-107. doi: https://doi.org/10.1080/01621459.1967.10482935.
Andersen, A., R., Snyder, L., H., Bradley, D., C., \& Xing, J. (1997). Multimodal representation of space in the posterior parietal cortex and its use in planning movements. Annual Review of Neuroscience, 20(1), 303-330. doi:https://doi.org/10.1146/annurev.neuro.20.1.303

Anderson, B. A., Laurent, P. A., \& Yantis, S. (2011). Value-driven attentional capture. Proceedings of the National Academy of Sciences, 108(25), 10367-10371. doi:https://doi.org/10.1073/pnas. 1104047108

Anderson, B. A., Laurent, P. A., \& Yantis, S. (2012). Generalization of value-based attentional priority. Visual Cognition, 20, 647-658.

Anderson, B. A. (2013). A value-driven mechanism of attentional selection. Journal of Vision, 13(3):7, 1-16.

Anderson, B. A. (2015). Value-driven attentional priority is context specific. Psychonomic Bulletin \& Review, 22(3), 750-756. doi:https:// doi.org/10.3758/s13423-014-0724-0

Anderson, B. A. (2016). The attention habit: How reward learning shapes attentional selection. Annals of the New York Academy of Sciences, 1369, 24-39.

Anderson, B. A., Kuwabara, H., Wong, D. F., Gean, E. G., Rahmim, A., Brasic, J. R., George, N., Frolov, B., Courtney, S. M., \& Yantis, S. (2016). The role of dopamine in value-based attentional orienting. Current Biology, 26, 550-555.

Anderson, B. A., Kuwabara, H., Wong, D. F., Roberts, J., Rahmim, A., Brasic, J. R., \& Courtney, S. M. (2017). Linking dopaminergic reward signals to the development of attentional bias: A positron emission tomographic study. NeuroImage, 157, 27-33.

Anderson, B. A., \& Halpern, M. (2017). On the value-dependence of value-driven attentional capture. Attention, Perception, \& Psychophysics, 79(4), 1001-1011. doi:https://doi.org/10.3758/ s13414-017-1289-6

Awh, E., Jonides, J., \& Reuter-Lorenz, P. A. (1998). Rehearsal in spatial working memory. Journal of Experimental Psychology: Human Perception and Performance, 24(3), 780-790. doi:https://doi.org/ 10.1037/0096-1523.24.3.780

Baddeley, A.D. (2007). Working memory, thought and action. Oxford, UK: Oxford Univ. Press. doi:https://doi.org/10.1093/acprof:oso/ 9780198528012.001.0001

Baddeley, A., Allen, R., \& Hitch, G. (2010). Investigating the episodic buffer. Psychologica Belgica, 50(3-4). doi:https://doi.org/10.5334/ pb-50-3-4-223

Barrouillet, P., Bernardin, S., \& Camos, V. (2004). Time constraints and resource sharing in adults' working memory spans. Journal of Experimental Psychology. General, 133(1), 83-100. doi:https:// doi.org/10.1037/0096-3445.133.1.83

Barrouillet, P., \& Camos, V. (2010). Working memory and executive control: A time-based resource-sharing account. Psychologica Belgica, 50(3-4). doi:https://doi.org/10.5334/pb-50-3-4-353

Benjamini, Y., \& Hochberg, Y. (1995). Controlling the false discovery rate: A practical and powerful approach to multiple testing. Journal of the Royal Statistical Society. Series B (Methodological), 57(1), 289-300.

Blacker, K. J., \& Courtney, S. M. (2016). Distinct neural substrates for maintaining locations and spatial relations in working memory. Frontiers in Human Neuroscience, 10. doi:https://doi.org/10.3389/ frhum.2016.00594

Blacker, K. J., Ikkai, A., Lakshmanan, B. M., Ewen, J. B., \& Courtney, S. M. (2016). The role of alpha oscillations in deriving and maintaining spatial relations in working memory. Cognitive, Affective, \& Behavioral Neuroscience, 16(5), 888-901. doi:https://doi.org/10. 3758/s13415-016-0439-y

Brainard, D. H. (1997). The psychophysics toolbox. Spatial Vision, 10(4), $433-436$.

Capotosto, P., Babiloni, C., Romani, G. L., \& Corbetta, M. (2009). Fronto-parietal cortex controls spatial attention through modulation of anticipatory alpha rhythms. The Journal of Neuroscience: The 
Official Journal of the Society for Neuroscience, 29(18), 58635872. doi:https://doi.org/10.1523/JNEUROSCI.0539-09.2009

Courtney, S. M. (2004). Attention and cognitive control as emergent properties of information representation in working memory. Cognitive, Affective, \& Behavioral Neuroscience, 4(4), 501-516. doi:https://doi.org/10.3758/CABN.4.4.501

D'Esposito, M., Postle, B. R., \& Rypma, B. (2000). Prefrontal cortical contributions to working memory: Evidence from event-related fMRI studies. Experimental Brain Research, 133(1), 3-11. doi: https://doi.org/10.1007/s002210000395

Eimer, M. (1996). The N2pc component as an indicator of attentional selectivity. Electroencephalogr. Clin. Neurophysiol. 99, 225-234.

Eimer, M., \& Kiss, M. (2008). Involuntary attentional capture is determined by task set: Evidence from event-related brain potentials. J. Cogn. Neurosci. 20, 1423-1433.

Faul, F., Erdfelder, E., Lang, A.-G., \& Buchner, A. (2007). G*Power 3: A flexible statistical power analysis program for the social, behavioral, and biomedical sciences. Behavior Research Methods, 39(2), 175191. doi:10.3758/BF03193146

Fougnie, D. (2008). The relationship between attention and working memory. In New research on short-term memory. NB Johansen (Ed.). Nova Science Publishers: New York.

Harris, A. M., Dux, P. E., Jones, C. N., \& Mattingley, J. B. (2017). Distinct roles of theta and alpha oscillations in the involuntary capture of goal-directed attention. NeuroImage, 152, 171-183. doi: https://doi.org/10.1016/j.neuroimage.2017.03.008

Hickey, C., McDonald, J.J., \& Theeuwes, J. (2006). Electrophysiological evidence of the capture of visual attention. J. Cogn. Neurosci. 18, 604-613.

Hinault, T., \& Lemaire, P. (2016). Adaptive strategic variations in human cognition across the life span. Journal of Education and Training, 3(1), 189-198. doi:https://doi.org/10.5296/jet.v3i1.8967

Hinault, T., \& Lemaire, P. (2017). Aging, rule-violation checking strategies, and strategy combination: An EEG study in arithmetic. International Journal of Psychophysiology, 120, 23-32. doi: https://doi.org/10.1016/j.ijpsycho.2017.07.003

Hinault, T., Lemaire, P., \& Phillips, N. (2016). Aging and sequential modulations of poorer strategy effects: An EEG study in arithmetic problem solving. Brain Research, 1630, 144-158. doi:https://doi. org/10.1016/j.brainres.2015.10.057

Ikkai, A., Blacker, K. J., Lakshmanan, B. M., Ewen, J. B., \& Courtney, S. M. (2014). Maintenance of relational information in working memory leads to suppression of the sensory cortex. Journal of Neurophysiology, 112(8), 1903-1915. doi:https://doi.org/10.1152/ jn.00134.2014

Itthipuripat, S., Cha, K., Rangsipat, N., \& Serences, J. T. (2015). Valuebased attentional capture influences context-dependent decisionmaking. Journal of Neurophysiology, 114(1), 560-569. doi:https:// doi.org/10.1152/jn.00343.2015

Jensen, O., Gelfand, J., Kounios, J., \& Lisman, J. E. (2002). Oscillations in the alpha band $(9-12 \mathrm{~Hz})$ increase with memory load during retention in a short-term memory task. Cerebral Cortex, 12(8), $877-882$.

Jensen O., Bonnefond M., \& VanRullen R. (2012). An oscillatory mechanism for prioritizing salient unattended stimuli. Trends Cogn Sci, 16, 200-206. doi:https://doi.org/10.1016/j.tics.2012.03.002

Johnston, W. A., Hawley, K. J., Plewe, S. H., Elliott, J. M. G., \& DeWitt, M. J. (1990). Attention capture by novel stimuli. Journal of Experimental Psychology: General, 119, 397-411.

Johnston, W. A., \& Schwarting, I. S. (1997). Novel popout: An enigma for conventional theories of attention. Journal of Experimental Psychology: Human Perception and Performance, 23, 622-631.

Jonides, J., \& Yantis, S. (1988). Uniqueness of abrupt visual onset in capturing attention. Perception \& Psychophysics, 43(4), 346-354. doi:https://doi.org/10.3758/BF03208805
Keehn, B., Westerfield, M., Müller, R.-A., \& Townsend, J. (2017). Autism, attention, and alpha oscillations: An electrophysiological study of attentional capture. Biological Psychiatry: Cognitive Neuroscience and Neuroimaging, 2(6), 528-536. doi:https://doi. org/10.1016/j.bpsc.2017.06.006

Klimesch, W. (1999). EEG alpha and theta oscillations reflect cognitive and memory performance: a review and analysis. Brain Research Reviews, 29(2-3), 169-195.

Klimesch, W., Sauseng, P., \& Hanslmayr, S. (2007). EEG alpha oscillations: The inhibition-timing hypothesis. Brain Research Reviews, 53(1), 63-88. doi:https://doi.org/10.1016/j.brainresrev.2006.06.003

Klimesch, W. (2012). Alpha-band oscillations, attention, and controlled access to stored information. Trends in Cognitive Sciences, 16(12), 606-617. doi:https://doi.org/10.1016/j.tics.2012.10.007

Kumar, S., Soto, D., \& Humphreys, G. W. (2009). Electrophysiological evidence for attentional guidance by the contents of working memory. European Journal of Neuroscience, 30(2), 307-317. doi:https:// doi.org/10.1111/j.1460-9568.2009.06805.x

Kumar, S., Higgs, S., Rutters, F., \& Humphreys, G. W. (2016). Biased towards food: Electrophysiological evidence for biased attention to food stimuli. Brain and Cognition, 110, 85-93. doi:https://oi.org/ 10.1016/j.bandc.2016.04.007

Le Pelley, M. E., Pearson, D., Porter, A., Yee, H., \& Luque, D. (2018). Oculomotor capture is influenced by expected reward value but (maybe) not predictiveness. Quarterly Journal of Experimental Psychology, 17470218.2017.1. doi:https://doi.org/10.1080/ 17470218.2017.1313874

Luck, S. J. (2012). Electrophysiological correlates of the focusing of attention within complex visual scenes: N2pc and related ERP components. In S. J. Luck \& E. S. Kappenman (Eds.), Oxford handbook of ERP components. New York: Oxford University Press.

Luck, S. J., \& Hillyard, S. A. (1994). Spatial filtering during visual search: Evidence from human electrophysiology. Journal of Experimental Psychology. Human Perception and Performance, 20(5), 1000-1014.

Luck, S. J., \& Ford, M. A. (1998). On the role of selective attention in visual perception. Proceedings of the National Academy of Sciences, 95, 825-830.

MacLean, M. H., \& Giesbrecht, B. (2015). Neural evidence reveals the rapid effects of reward history on selective attention. Brain Research, 1606, 86-94. doi:https://doi.org/10.1016/j.brainres.2015. 02.016

Maddux, J.-M., Kerfoot, E. C., Chatterjee, S., \& Holland, P. C. (2007). Dissociation of attention in learning and action: Effects of lesions of the amygdala central nucleus, medial prefrontal cortex, and posterior parietal cortex. Behavioral Neuroscience, 121(1), 63-79. doi:https:// doi.org/10.1037/0735-7044.121.1.63

Makovski, T., Swallow, K. M., \& Jiang, Y. V. (2011). Attending to unrelated targets boosts short-term memory for color arrays. Neuropsychologia, 49(6), 1498-1505. doi:https://doi.org/10.1016/ j.neuropsychologia.2010.11.029

Marchner, J. R., \& Preuschhof, C. (2018). Reward history but not search history explains value-driven attentional capture. Attention, Perception \& Psychophysics. doi:https://doi.org/10.3758/s13414018-1513-Z

Maris, E., \& Oostenveld, R. (2007). Nonparametric statistical testing of EEG- and MEG-data. Journal of Neuroscience Methods, 164(1), 177-190. doi:https://doi.org/10.1016/j.jneumeth.2007.03.024

Mazza, V., Turatto, M., \& Caramazza, A. (2009). Attention selection, distractor suppression and N2pc. Cortex, 45(7), 879-890. doi: https://doi.org/10.1016/j.cortex.2008.10.009

Miller, G. A. (1956). The magical number seven, plus or minus two: Some limits on our capacity for processing information. Psychological Review, 63(2), 81-97. doi:https://doi.org/10.1037/ h0043158 
Nee, D. E., Brown, J. W., Askren, M. K., Berman, M. G., Demiralp, E., Krawitz, A., \& Jonides, J. (2013). A meta-analysis of executive components of working memory. Cerebral Cortex, 23(2), 264 282. doi:https://doi.org/10.1093/cercor/bhs007

Neo, G., \& Chua, F. K. (2006). Capturing focused attention. Perception and Psychophysics, 68, 1286-1296.

Noonan, M. P., Adamian, N., Pike, A., Printzlau, F., Crittenden, B. M., \& Stokes, M. G. (2016). Distinct mechanisms for distractor suppression and target facilitation. The Journal of Neuroscience, 36(6), 1797-1807. doi:https://doi.org/10.1523/JNEUROSCI.2133-15. 2016

Oostenveld, R., Fries, P., Maris, E., \& Schoffelen, J.-M. (2011). FieldTrip: Open source software for advanced analysis of MEG, EEG, and invasive electrophysiological data. Computational Intelligence and Neuroscience, 2011, 1-9. doi:https://doi.org/10. $1155 / 2011 / 156869$

Pelli, D. G. (1997). The VideoToolbox software for visual psychophysics: Transforming numbers into movies. Spatial Vision, 10(4), 437 442.

Postle, B.,R., \& Hamidi, M. (2007). Nonvisual codes and nonvisual brain areas support visual working memory. Cerebral Cortex, 17, 21512162. doi:https://doi.org/10.1093/cercor/bhl123

Qi, S., Zeng, Q., Ding, C., \& Li, H. (2013). Neural correlates of rewarddriven attentional capture in visual search. Brain Research, 1532, 32-43. doi:https://doi.org/10.1016/j.brainres.2013.07.044

Rutherford, H. J. V., O'Brien, J. L., \& Raymond, J. E. (2010). Value associations of irrelevant stimuli modify rapid visual orienting. Psychonomic Bulletin \& Review, 17(4), 536-542. doi:https://doi. org/10.3758/PBR.17.4.536

Sala, J.B., \& Courtney, S. M. (2009). Flexible working memory representations of the relationships between an object and its location as revealed by interactions with attention. Attention, Perception, \& Psychophysics, 71(7), 1525-33. doi:https://doi.org/10.3758/APP. 71.7.1525.

Sawaki, R., \& Luck, S. J. (2013). Active suppression after involuntary capture of attention. Psychonomic Bulletin \& Review, 20(2), 296301. doi:https://doi.org/10.3758/s13423-012-0353-4

Sokhadze, E. M., El-Baz, A., Baruth, J., Mathai, G., Sears, L., \& Casanova, M. F. (2009). Effects of low frequency repetitive transcranial magnetic stimulation (rTMS) on gamma frequency oscillations and event-related potentials during processing of illusory figures in autism. Journal of Autism and Developmental Disorders, 39(4), 619-634. doi:https://doi.org/10.1007/s10803-008-0662-7

Sha, L. Z., \& Jiang, Y. V. (2016). Components of reward-driven attentional capture. Attention, Perception, \& Psychophysics, 78(2), 403414. doi:https://doi.org/10.3758/s13414-015-1038-7

Tadel, F., Baillet, S., Mosher, J. C., Pantazis, D., \& Leahy, R. M. (2011). Brainstorm: A user-friendly application for MEG/EEG analysis. Computational Intelligence and Neuroscience, 2011, 1-13. doi: https://doi.org/10.1155/2011/879716

Töllner, T., Müller, H. J., \& Zehetleitner, M. (2012). Top-down dimensional weight set determines the capture of visual attention: Evidence from the PCN component. Cerebral Cortex, 22 (7), 1554-1563.

Unsworth, N., \& Robison, M. K. (2016). The influence of lapses of attention on working memory capacity. Memory \& Cognition, 44(2), 188-196. doi:https://doi.org/10.3758/s13421-015-0560-0

van Diepen, R. M., Miller, L. M., Mazaheri, A., \& Geng, J. J. (2016). The role of alpha activity in spatial and feature-based attention. eNeuro, 3(5), ENEURO.0204-16.2016. doi:https://doi.org/10.1523/ ENEURO.0204-16.2016

van Dijk, H., van der Werf, J., Mazaheri, A., Medendorp, W. P., \& Jensen, O. (2010). Modulations in oscillatory activity with amplitude asymmetry can produce cognitively relevant event-related responses. Proceedings of the National Academy of Sciences, 107(2), 900 905. doi:https://doi.org/10.1073/pnas.0908821107

Vandierendonck, A. (2016). A working memory system with distributed executive control. Perspectives on Psychological Science, 11(1), 74-100. doi:https://doi.org/10.1177/1745691615596790

Woodman, G. F., \& Luck, S. J. (1999). Electrophysiological measurement of rapid shifts of attention during visual search. Nature, 400: 867-869.

Wykowska, A., \& Schubö, A. (2009). On the temporal relation of topdown and bottom-up mechanisms during guidance of attention. Journal of Cognitive Neuroscience, 22(4), 640-654. doi:https:// doi.org/10.1162/jocn.2009.21222 